\title{
Storage of Heat, Cold and Electricity
}

\author{
Anastasia Stamatiou, Andreas Ammann, Andreas Abdon, Ludger J. Fischer, Damian Gwerder, \\ and Jörg Worlitschek ${ }^{\star}$
}

\begin{abstract}
A promising energy storage system is presented based on the combination of a heat pump, a heat engine, a hot and a cold storage. It can be operated as a pure bulk electricity storage (alternative to Pumped Heat Electrical Storage (PHES)/ Compressed Air Energy Storage (CAES)) or as combined storage of heat, cold and electricity. Both variations have been evaluated using a steady state, thermodynamic model and two promising concepts are proposed: A transcritical $\mathrm{CO}_{2}$ cycle for the pure electricity storage and a subcritical $\mathrm{NH}_{3}$ cycle for combined storage of electricity, heat and cold. Parametric studies are used to evaluate the influence of different parameters on the roundtrip efficiency of the storage system.
\end{abstract}

Keywords: Dual Energy Storage \& Converter · Electricity storage $\cdot$ Power-to-Heat $\cdot$ Reversible heat pump

\section{Introduction}

Storage technologies are bound to play an important role in the integration of renewable energy sources and in increasing the energy utilization efficiency in the upcoming years. The development of efficient and cost-effective storage technologies in all energy sectors is crucial to this task. ${ }^{[1]}$ Along with the development of innovative electricity, thermal and chemical storage, the establishment of systems that can provide flexibility between energy forms will also gain importance. [2]

A promising storage system based on the combination of a heat pump, a heat engine, a cold and a hot storage is presented (Fig. 1). The system can be designed to serve as pure bulk electricity storage or a combination of heat, cold and electricity storage. The underlining operating principle is that during periods of excess electricity generation, a heat pump is operated to charge a hot and in some cases also a cold storage. In periods of excess electricity demand, the system can be operated in reverse as a heat engine, using the stored

${ }^{\star}$ Correspondence: Prof. Dr. J. Worlitschek Group Thermal Energy Storage (TES)

Competence Centre Thermal Energy Systems \& Process Engineering

Lucerne University of Applied Sciences and Arts $\mathrm{CH}-6048$ Horw, Switzerland

E-mail: joerg.worlitschek@hslu.ch temperature difference to generate electricity. This system variation is particularly interesting for bulk electricity storage in a power output range higher than $10 \mathrm{MW}$. It can be viewed as an alternative to Pumpedstorage Hydroelectricity (PSH or PHES) and Compressed Air Electricity Storage (CAES), with the additional advantages of a higher energy density and the possibility of site-independent installation.

The first reports of this type of storage system can be found as early as $1924^{[3]}$ and were continued in the $1970 \mathrm{~s}^{[4]}$ under the term 'reversible heat pumping'. In recent years, this technology has been the subject of several independent academic and industrial investigations with varying system specifications (working fluid, temperature levels, storing material, etc.). Most investigators named the system differently (e.g. ETES, ${ }^{[5]}$ PTES, ${ }^{[6]}$ CHEST, ${ }^{[7]}$ TEES, ${ }^{[8]}$ and others ${ }^{[9]}$ ), making the identification of relevant projects challenging.
The authors propose a thermally open variation of the technology, named Dual Energy Storage \& Converter, where the two storage units can be used, not only as a means to store electricity but also to cover heating and cooling demands. This version offers additional flexibility between energy forms (power, heat and cold) and can be implemented in applications which possess a relatively high demand of all three energy forms during the whole year (e.g. food \& beverages industry). A thermally open variation of the reversible heat pump has been previously considered for building applications using water storage for the high temperature side but no storage on the low temperature side. ${ }^{[10]}$

This work presents the evaluation process of different variations of the storage system by means of a simple thermodynamic tool. Two promising systems are selected and are shown in detail in terms of temperature-entropy diagrams.

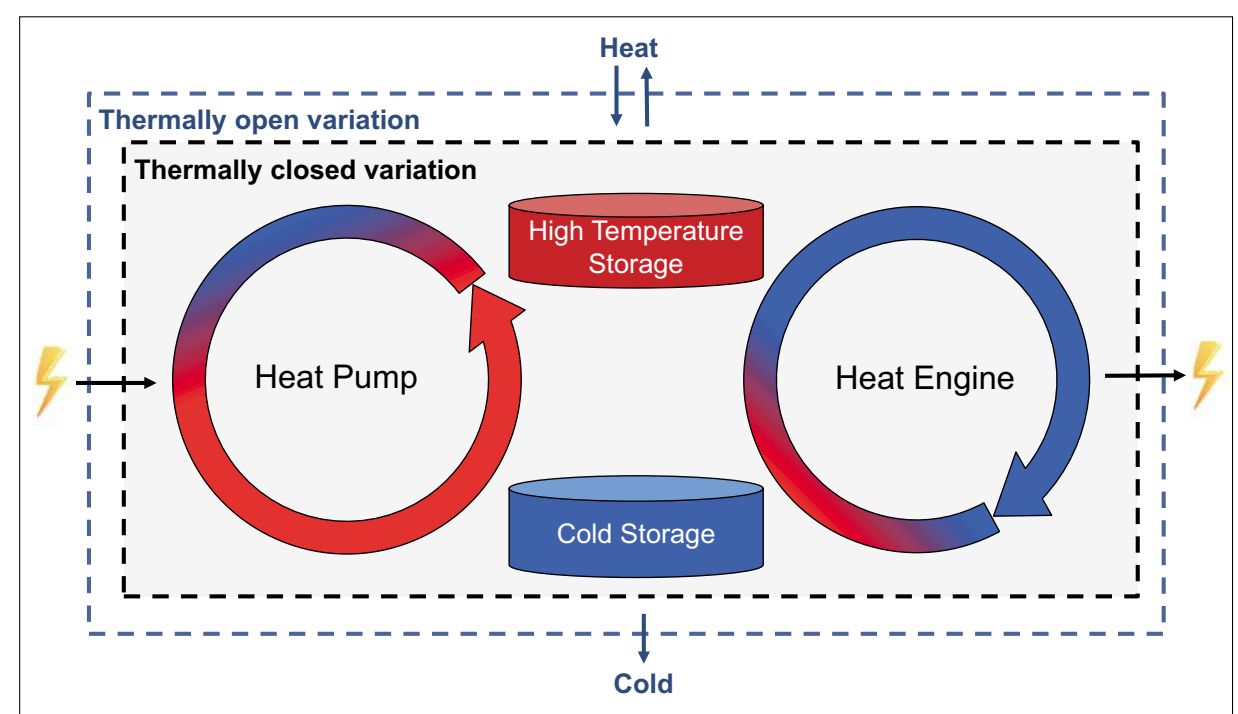

Fig. 1. Schematic of the DESC process comprising a heat pump, a heat engine, a hot and a cold storage. 


\section{Methods}

A simple steady-state thermodynamic tool has been developed for the scanning of different variations of the storage system shown in Fig. 1. The tool is based on Matlab $^{[11]}$ and extracts the thermodynamic properties from the NIST-REFPROP database. ${ }^{[12]}$ It calculates the thermodynamic points describing the basic processes taking place during the heat pump and Rankine cycle (e.g. evaporation, expansion, condensation, etc.). Both the inefficiencies introduced by the machines and the exergy losses during heat exchange are considered but pressure drops and heat losses are neglected. The model was verified using independently calculated values describing the $\mathrm{ABB}$ concept with transcritical $\mathrm{CO}_{2}$. It was subsequently used to carry out parametric studies of the systems considered and assess variations of the technology (working fluids, number of stages, etc.).

\section{Results and Discussion}

The main characteristics of the two system variations selected to be presented in this work are shown in Table 1. ETES is a bulk electricity storage concept developed by $\mathrm{ABB}^{[5]}$ while DESC is a combined heat, cold and electricity storage concept developed by the authors.

The first thermodynamic evaluation was performed for the ETES system and can be seen in Fig. 2. The concept is based on transcritical $\mathrm{CO}_{2}$ as working fluid for thermally closed cycles (see Table 1 ). The red line represents the heat pump cycle, the blue the Rankine cycle. Water is used for the sensible high temperature storage at $120{ }^{\circ} \mathrm{C}$ whereas ice is used for the low temperature storage at $0{ }^{\circ} \mathrm{C}$. As seen in Fig. 2, the maximum operating pressure is 136.3 bar. The temperature levels and storage units are chosen to create an optimum match between the condensation/evaporation processes of the working fluid and the behavior of the storage media (water/ice) during charging/discharging. The ability to utilize water as the storage medium on both sides represents one of the biggest advantages of this variation of the reversible heat pump concept. The model was verified using independently calculated values provided by $\mathrm{ABB}$. After the verification, the model was used to carry out parametric studies examining the influence of different parameters on the roundtrip efficiency of ETES. The parameters considered were: (i) temperature of hot storage, (ii) temperature of cold storage, (iii) ambient temperature, (iv) $\Delta T$ during heat transfer and (v) isentropic efficiencies of the mechanical components. Fig. 3 presents the results of
Table 1. Characteristics of technology variations presented in Figs 1-4

\begin{tabular}{|l|l|l|l|l|l|l|}
\hline Name & \multicolumn{2}{|l|}{ Energy form } & $\begin{array}{l}\text { Working } \\
\text { fluid }\end{array}$ & $\begin{array}{l}\text { Storage } \\
\text { units (hot/ } \\
\text { cold) }\end{array}$ & $\begin{array}{l}\text { Criteria for } \\
\text { choice of T levels }\end{array}$ & Application \\
\hline ETES & El. & El. & $\mathrm{CO}_{2}$ & $\begin{array}{l}\text { Sensible / } \\
\text { Latent (ice) }\end{array}$ & $\begin{array}{l}\text { High roundtrip } \\
\text { efficiency; } \\
\text { water as storage } \\
\text { material }\end{array}$ & $\begin{array}{l}\text { Bulk } \\
\text { electricity } \\
\text { storage }\end{array}$ \\
\hline DESC & $\begin{array}{l}\text { El, } \\
\text { heat }\end{array}$ & $\begin{array}{l}\text { El., } \\
\text { heat, } \\
\text { cold }\end{array}$ & $\mathrm{NH}_{3}$ & $\begin{array}{l}\text { Latent / } \\
\text { Latent }\end{array}$ & $\begin{array}{l}\text { Optimized for } \\
\text { customer-relevant } \\
\text { T levels }\end{array}$ & $\begin{array}{l}\text { Heat, cold, } \\
\text { electricity } \\
\text { and storage, } \\
\text { and supply }\end{array}$ \\
\hline
\end{tabular}

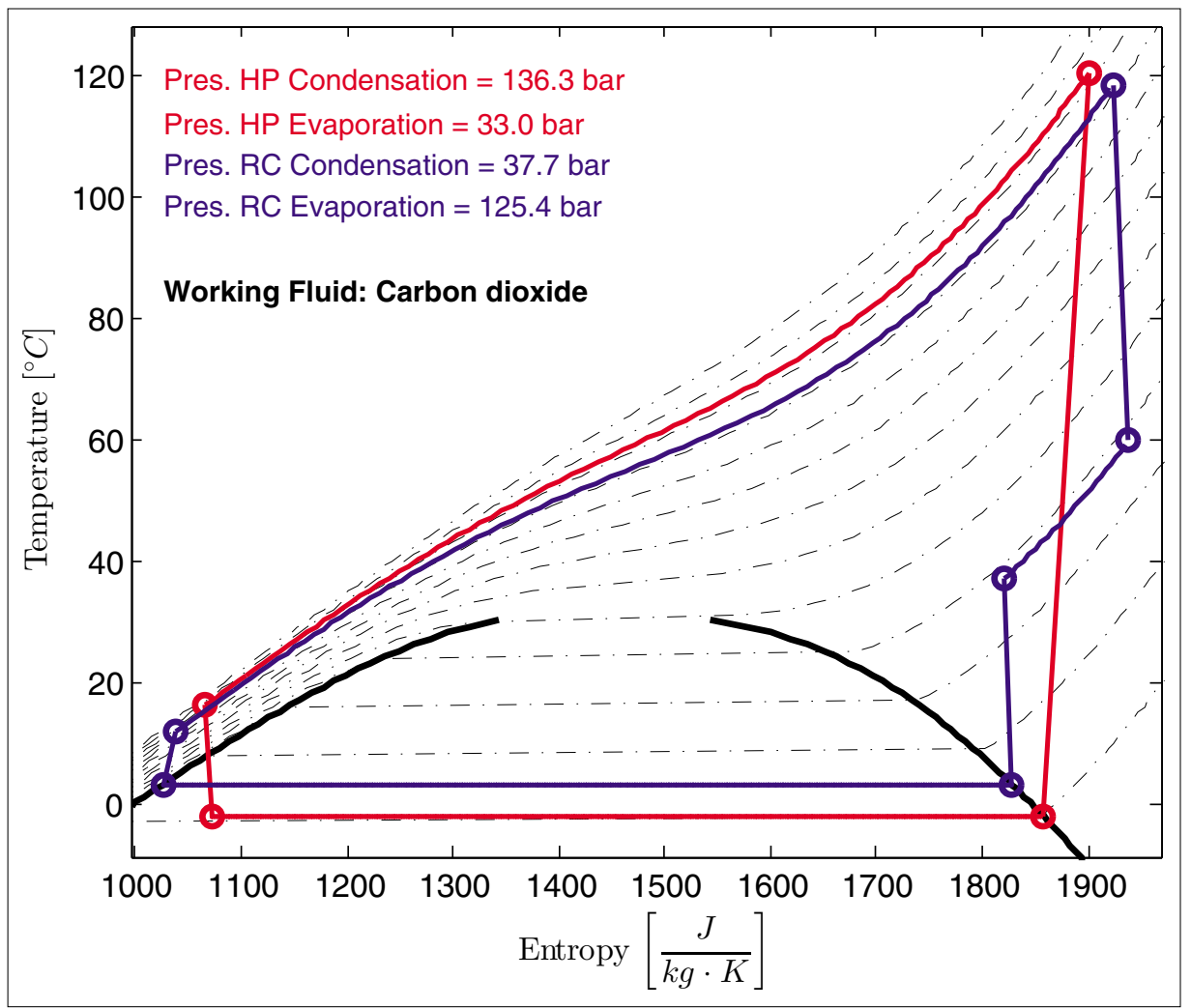

Fig. 2. Output of thermodynamic modeling for ETES concept with transcritical $\mathrm{CO}_{2}$ as described in Table 1.

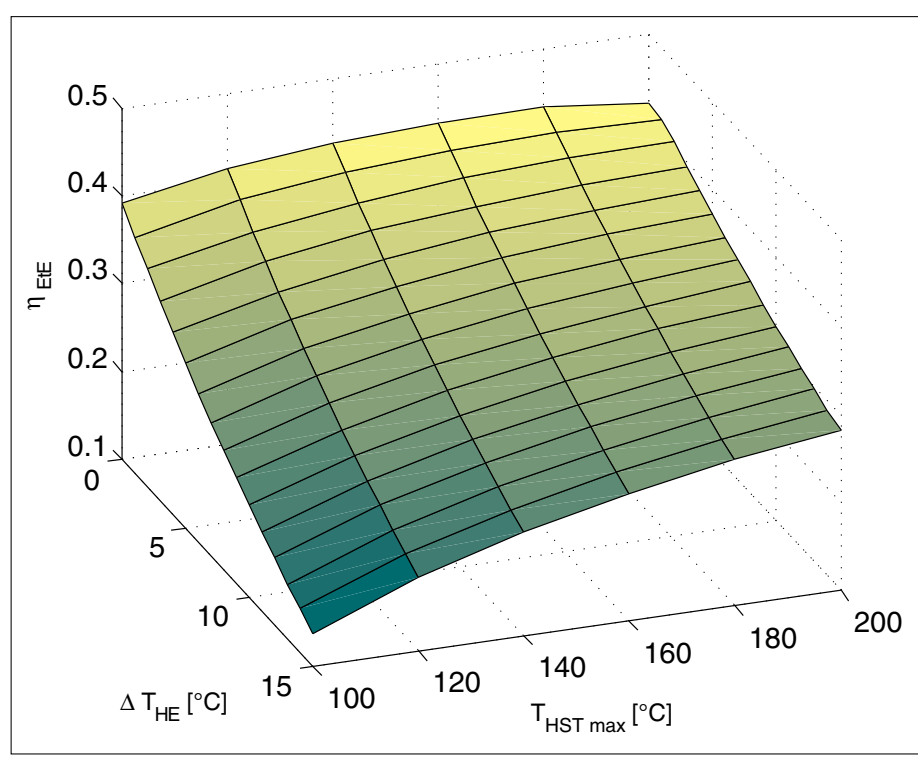

Fig. 3. Surface plot showing the roundtrip efficiency of the transcritical $\mathrm{CO}_{2}$ system, $\eta_{\mathrm{EtE}}$, as a function of the $\Delta T$ during the heat exchange and the maximum temperature of the high temperature storage $\mathrm{T}_{\text {HST }}$ 
one parametric analysis showing the evolution of the ETES roundtrip efficiency as a function of the maximum temperature of the hot storage and the $\Delta T$ during heat transfer. As expected, an increase in the heat exchanger $\Delta T$ results in a dramatic decrease in the ETES roundtrip efficiency $\eta_{E t E}$. In contrast, an increase in the maximum temperature of the hot storage results in an increase in the $\eta_{E t E}$.

The thermodynamic model was modified to describe the behavior of other reversible heat pump variations. Other working fluids were evaluated such as isobutane and ammonia. A promising variation for the combined storage uses $\mathrm{NH}_{3}$ as a working fluid (Fig. 4). Similarly to Fig. 2, the red line describes the heat pump cycle and the blue line the Rankine cycle. Both processes comprise two stages providing the flexibility of charging/discharging the hot and the cold storage separately. Both the produced heat and cold will be stored in latent heat storage units to ensure an optimal match with the temperature profile of the subcritical cycles. The storage temperatures have been chosen at $0{ }^{\circ} \mathrm{C}$ and $95^{\circ} \mathrm{C}$ because they represent two key temperature levels for industrial process heat and cold. Despite its toxicity, ammonia is a promising natural refrigerant with a very high enthalpy of vaporization. It has been used widely in refrigeration and there are already commercially available heat pumps that can deliver the temperatures required for this DESC variation. One big disadvantage is the high overheating during the heat pump operation which imposes challenges for the lubricant and induces mechanical stress on the components. The expansion processes during the heat engine mode take place in the two-phase region which imposes constraints on the choice of the expander. At the maximum operating pressure of $60 \mathrm{bar}$, even though not as high as the one demanded by the transcritical $\mathrm{CO}_{2}$ cycle, it could still impose challenges in the component development and operation.

\section{Conclusions}

A promising energy storage system is presented. It can be operated as a pure bulk electricity storage (alternative to PHES/CAES) or as combined storage of heat, cold and electricity. Both variations have been evaluated using a steady state, thermodynamic model and two promising concepts are being proposed: A transcritical $\mathrm{CO}_{2}$ cycle for the pure electricity stor-

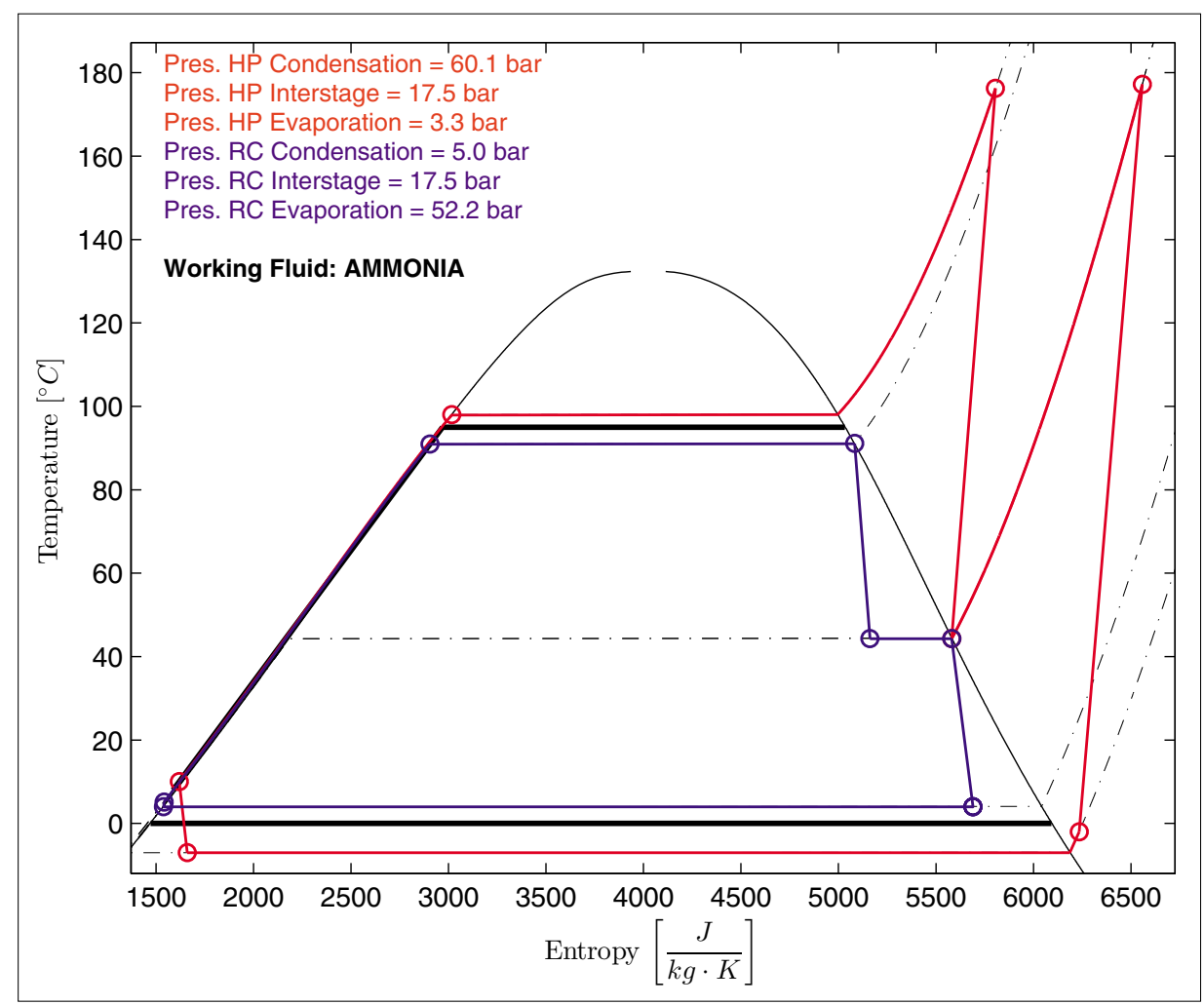

Fig. 4. Results of thermodynamic evaluation for DESC concept with subcritical $\mathrm{NH}_{3}$ as described in Table 1.

age and a subcritical $\mathrm{NH}_{3}$ cycle for combined storage of electricity, heat and cold. The $\mathrm{CO}_{2}$ system has a maximum operating temperature of $120{ }^{\circ} \mathrm{C}$ and can be combined with water as a storage medium on both the hot and cold sides, which offers a valuable simplicity to the ETES system. The $\mathrm{NH}_{3}$-based DESC system on the other hand can be integrated in a large number of industrial applications which require cold at $0{ }^{\circ} \mathrm{C}$ and heat at $\sim 95{ }^{\circ} \mathrm{C}$. The high heat of vaporisation of $\mathrm{NH}_{3}$ and high energy density of the latent heat storage units increase system compactness. The developed steady-state thermodynamic tool is suitable for a first scanning/evaluation of different storage systems variations. It can also be successfully used to perform parametric studies and find optimal operation points. For a more detailed estimation of the system potential, the tool has to be extended to capture transient phenomena and to include pressure drops and heat losses.

\section{Acknowledgments}

The authors thank the CTI (Commission for Technology and Innovation) for the financial support through SCCER Hae and ABB for providing data and support for the thermodynamic modelling of the ETES process.
[1] a) IEA, International Energy Agency 2014, 64; b) A. Hauer, 'Thermal Energy Storage - Technology Brief', International Energy Agency-Energy Technology Systems Analysis Programme IEA-ETSAP, International Renewable Energy Agency IRENA, 2013; c) D. Lindley, Nature News 2010, 463, 18.

[2] N. Tanaka, International Energy Agency Paris, France, 2011.

[3] F. Marguerre, Mitteilungen der Vereinigung der Elektrizitätswerke 1924, 354, 27e35.

[4] R. P. Cahn, 'Thermal energy storage by means of reversible heat pumping', Google Patents, 1978.

[5] M. Mercangöz, J. Hemrle, L. Kaufmann, A. Z'Graggen, C. Ohler, Energy 2012, 45, 407.

[6] A. White, G. Parks, C. N. Markides, Appl. Therm. Eng. 2013, 53, 291.

[7] W. Steinmann, Energy 2014, 69, 543.

[8] Y.-M. Kim, D.-G. Shin, S.-Y. Lee, D. Favrat, Energy 2013, 49, 484.

[9] T. Desrues, J. Ruer, P. Marty, J. F. Fourmigué, Appl. Therm. Eng. 2010, 30, 425.

[10] a) O. Dumont, S. Quoilin, V. Lemort, Int. J. Refrig. 2015, 54, 190; b) S. Schimpf, R. Span, Ener. Conv. Manag. 2015, 94, 430.

[11] Matlab and Statistics Toolbox, The MathWorks Inc., 2014, Massachusetts, USA.

[12] E. Lemmon, M. Huber, M. McLinden, 'NIST Reference Fluid Thermodynamic and Transport Properties - REFPROP', National Institute of Standards and Technology, 2010, Boulder, USA. 